\title{
Genes for asparagine metabolism in Lotus japonicus: differential expression and interconnection with photorespiration
}

\author{
Margarita García-Calderón ${ }^{\dagger}$, Carmen M. Pérez-Delgado ${ }^{\dagger}$, Alfredo Credali ${ }^{\dagger}$, José M. Vega, Marco Betti ${ }^{*}$ (D)
} and Antonio J. Márquez

\begin{abstract}
Background: Asparagine is a very important nitrogen transport and storage compound in plants due to its high nitrogen/carbon ratio and stability. Asparagine intracellular concentration depends on a balance between asparagine biosynthesis and degradation. The main enzymes involved in asparagine metabolism are asparagine synthetase (ASN), asparaginase (NSE) and serine-glyoxylate aminotransferase (SGAT). The study of the genes encoding for these enzymes in the model legume Lotus japonicus is of particular interest since it has been proposed that asparagine is the principal molecule used to transport reduced nitrogen within the plant in most temperate legumes.

Results: A differential expression of genes encoding for several enzymes involved in asparagine metabolism was detected in L. japonicus. ASN is encoded by three genes, LjASN1 was the most highly expressed in mature leaves while LjASN2 expression was negligible and LjASN3 showed a low expression in this organ, suggesting that LJASN1 is the main gene responsible for asparagine synthesis in mature leaves. In young leaves, LjASN3 was the only ASN gene expressed although at low levels, while all the three genes encoding for NSE were highly expressed, especially LjNSE1. In nodules, LjASN2 and LjNSE2 were the most highly expressed genes, suggesting an important role for these genes in this organ. Several lines of evidence support the connection between asparagine metabolic genes and photorespiration in $L$. japonicus: a) a mutant plant deficient in LjNSE1 showed a dramatic decrease in the expression of the two genes encoding for SGAT; b) expression of the genes involved in asparagine metabolism is altered in a photorespiratory mutant lacking plastidic glutamine synthetase; c) a clustering analysis indicated a similar pattern of expression among several genes involved in photorespiratory and asparagine metabolism, indicating a clear link between LjASN1 and LjSGAT genes and photorespiration.
\end{abstract}

Conclusions: The results obtained in this paper indicate the existence of a differential expression of asparagine metabolic genes in L. japonicus and point out the crucial relevance of particular genes in different organs. Moreover, the data presented establish clear links between asparagine and photorespiratory metabolic genes in this plant.

Keywords: Asparaginase genes, Asparagine synthetase genes, Lotus japonicus, Serine-glyoxylate aminotransferase genes

\footnotetext{
* Correspondence: mbetti@us.es

${ }^{\dagger}$ Equal contributors

Departamento de Bioquímica Vegetal y Biología Molecular, Facultad de

Química, C/ Profesor García González, 1, 41012 Sevilla, Spain
}

(c) The Author(s). 2017 Open Access This article is distributed under the terms of the Creative Commons Attribution 4.0 International License (http://creativecommons.org/licenses/by/4.0/), which permits unrestricted use, distribution, and reproduction in any medium, provided you give appropriate credit to the original author(s) and the source, provide a link to the Creative Commons license, and indicate if changes were made. The Creative Commons Public Domain Dedication waiver (http://creativecommons.org/publicdomain/zero/1.0/) applies to the data made available in this article, unless otherwise stated. 


\section{Background}

Asparagine is a very important nitrogen transport and storage compound in plants due to its high nitrogen/carbon ratio and stability. In fact, asparagine is the major nitrogen transport compound in the xylem from the root to the leaves and in the phloem from the leaves to the developing seed in a range of plants, particularly in temperate legumes [1]. This is the case of the model legume Lotus japonicus, where it was shown that asparagine can account for $86 \%$ of the nitrogen flux from root to shoot when nitrogen is not limiting [2].

Asparagine intracellular concentration depends on a balance between asparagine biosynthesis and degradation. The main route for asparagine biosynthesis in plants is mediated by the enzyme asparagine synthetase (ASN, EC 6.3.5.4), which catalyzes the ATP-dependent transfer of the amide group of glutamine to aspartate yielding asparagine in the presence of magnesium. Most plant species contain two or more ASN genes mainly grouped into two phylogenetic clades, named class-I and class-II [3, 4], whose expression is subjected to metabolic control. Class-I genes are usually negatively regulated by light or sugars whereas class-II genes are not repressed by light $[5,6]$. Besides light and sugars, nitrogen source and availability are other factors that regulate ASN expression [6-8]. This fine regulation allows the plants to have asparagine available at specific phases of development, such as nitrogen mobilization in germinating seeds, ammonium (re)assimilation in leaves and nitrogen remobilization from senescent leaves to developing seeds $[3,9,10]$. The role of a specific asparagine synthetase isoform in primary ammonium assimilation has been also recently investigated [11].

On the other hand, two pathways for asparagine catabolism have been established in plants. The main enzyme for asparagine degradation is asparaginase (NSE, EC 3.5.1.1) that catalyzes the hydrolysis of asparagine to yield aspartate and ammonia, which is subsequently reassimilated by glutamine synthetase [12-15]. The other asparagine degradation route involves the transamination of asparagine in the presence of glyoxylate to yield 2oxosuccinamate and glycine, catalyzed by asparagineglyoxylate aminotransferase [16].

Two subfamilies of asparaginases have been described, corresponding to biochemical subtypes defined on the basis of their dependence for $\mathrm{K}^{+}$: $\mathrm{K}^{+}$-dependent and $\mathrm{K}$ ${ }^{+}$-independent asparaginases [13, 14, 17, 18]. The exact meaning of the potassium dependence in asparaginases constitutes an interesting topic of research $[14,19]$. Despite of their separate classification, the two groups of plant asparaginases share significant levels (about 60\%) of sequence identity.

Asparagine aminotransferase appears to be the same protein as the peroxisomal photorespiratory enzyme serine-glyoxylate aminotransferase (SGAT, EC 2.6.1.45), based on its substrate preference and subcellular localization [16, 20-24]. The 2-oxosuccinamate produced by this enzyme is then reduced to hydroxysuccinamate and subsequently deamidated to yield malate or, alternatively, to oxalacetate and ammonia, by the enzyme omega-amidase [25]. In plants, asparagine transamination catalyzed by SGAT has been proposed to be involved in photorespiration as an input of external nitrogen into the photorespiratory cycle [25-27]. However, SGAT enzymes have been also shown to use serine or alanine efficiently as substrate, besides asparagine [16].

In the present paper the pattern of expression of asparagine metabolism genes was examined in different organs and under different conditions (e.g. light and dark) in L. japonicus plants. In addition, the connection between asparagine metabolism genes and photorespiration was also analyzed using two different mutants from this plant. On the one hand, a mutant deficient in asparaginase, called Ljnse1-4, which accumulates high levels of asparagine [15]. On the other hand, a photorespiratory mutant called Ljgln2-2, deficient in the plastidic isoform of glutamine synthetase (GS2), which is the enzyme that reassimilates the $\mathrm{NH}_{4}^{+}$produced by the photorespiratory cycle $[28,29]$. The expression levels of different genes involved in asparagine metabolism and in the photorespiratory pathway were determined by quantitative RT-PCR in leaves using WT (wild type) and both mutant genotypes. A global transcriptomic analysis was also carried out to further investigate the interconnection between asparagine metabolism and photorespiratory metabolism genes in this plant. The data presented in this paper indicate the existence of a differential expression of asparagine metabolism genes in L. japonicus and point out the crucial relevance of particular genes in different organs and/or processes related with asparagine metabolism and the photorespiratory pathway in this plant.

\section{Methods}

\section{Plant materials and treatments}

L. japonicus (Regel) Larsen cv. Gifu was initially obtained from Prof. Jens Stougaard (University of Aarhus, Denmark) and then self-propagated at the University of Seville. Plant seeds were scarified and surface-sterilized, germinated in Agar/Horned Petri dishes, and transferred to pots using vermiculite as solid support. Five seedlings were placed in each pot and grown during 35 days in a chamber under $16 / 8 \mathrm{~h}$ day/night, $20 / 18{ }^{\circ} \mathrm{C}$, with a photosynthetic photon flux density of $250 \mu \mathrm{mol} \mathrm{m}{ }^{-2} \cdot \mathrm{s}^{-1}$ and a constant humidity of $70 \%$. Plants were watered with Hornum nutrient solution [30]. Plants grown in symbiosis with nitrogen-fixing bacteria were inoculated with Mesorhizobium loti, as described previously by García-Calderón et al. [31]. 
The Ljgln2-2 mutant used in this work was previously isolated from a photorespiratory mutant screening $[28,32,33]$. The mutant offspring of two consecutive backcrosses of Ljgln2-2 into the WT background was employed. In addition, for other experiments, a mutant in the LjNSE1 gene of L. japonicus was used, called Ljnse1-4, that was previously isolated by TILLING (Targeted Induced Local Lesions IN Genomes) as described by Credali et al. [15].

\section{RNA extraction and qRT-PCR}

Tissues from $L$. japonicus plants were harvested and immediately frozen in liquid nitrogen, homogenized with a mortar and pestle, and kept at $-80{ }^{\circ} \mathrm{C}$ until use. Total RNA was isolated using the hot borate method [34]. The integrity and concentration of the RNA preparations were checked using an Experion bioanalyzer (Bio-Rad) with RNA StdSens chips and a NanoDrop ND-1000 (NanoDrop Technologies), respectively. RNA extraction was carried out using three independent biological samples for each genotype/condition/tissue. A biological replicate consisted of tissue pooled from five plants grown in the same pot.

For qRT-PCR analysis, total RNA was treated with the TURBO DNA-free Kit (Ambion). Reverse transcription was carried out using SuperScript III reverse transcriptase (Invitrogen), oligo-dT and RNAsin RNAse inhibitor (Promega). DNA contamination and RNA integrity were checked by performing the qRT-PCR reactions with oligonucleotides that amplified an intron in the L. japonicus hypernodulation aberrant root formation (LjHAR1) gene and the $5^{\prime}$ and $3^{\prime}$ ends of the L. japonicus glyceraldehyde-3-phosphate dehydrogenase respectively (using the oligonucleotide pairs LjGAPDH5' and LjGAPDH3'). qRT-PCR analysis was performed as described by Pérez-Delgado et al. [35] using the SensiFAST $^{\mathrm{Ts}}$ SYBR $^{\oplus}$ No-ROX Kit (Bioline) and a LightCycler ${ }^{\oplus}$ 480 II thermal cycler (Roche).

$\mathrm{Ct}$ values were determined using the LightCycler 480 software version 1.5 .0 and the $2^{-\mathrm{Ct}}$ values were standardized by dividing them by geometric mean of the $2^{-\mathrm{Ct}}$ values of four different housekeeping genes: L. japonicus glycosylphosphatidyl inositol $(L j G P I)$-anchored protein (Lj3g3v1933150.1), L. japonicus protein phosphatase 2A (LjPp2A) (Lj2g3v0742070.1), L. japonicus ubiquitin carrier protein $10(\mathrm{LjUbc10})(\mathrm{Lj} 1 \mathrm{~g} 3 \mathrm{v} 2063210.1)$ and $L$. japonicus polyubiquitin 4 (LjUbq) (Lj5g3v2060710.1) that were selected among the most stably expressed genes in plants [36], and that were found to be suitable for gene expression analysis also in L. japonicus [34, 35, 37]. A list of all oligonucleotides used is provided in Additional file 1: Table S1.

\section{Clustering analysis of qRT-PCR data}

For hierarchical clustering of qRT-PCR data the transcript levels of WT plants under high $\mathrm{CO}_{2}$ conditions were taken as 1 and the differences between the $\log _{2}$ of relative expression levels of $\operatorname{Ljg} \ln 2-2$ and WT are presented. Hierarchical clustering of the data was performed using the Multiexperiment Viewer software version 4.9.0 [38] with optimized gene leaf order and complete linkage clustering algorithm. The expression data of some genes of nitrogen and photorespiratory metabolism were taken from Pérez-Delgado et al. [39].

\section{Clustering analysis of microarray data}

\section{a). Microarray data collection and preprocessing}

The microarray data used in this work were obtained from the experiments published by: Sánchez et al. [34], Pérez-Delgado et al. [39], Høgslund et al. [40], Díaz et al. [41], Sánchez et al. [42], and Betti et al. [43]. These experiments have a total of 84 different conditions (samples) and 240 hybridizations. CEL files of these experiments are available in the public microarrays database ArrayExpress [44]. The code numbers of the experiments are: E-MEXP1204, E-TABM-715, E-MEXP-2344, E-MEXP-2690, EMEXP-1726, E-MEXP-3710 and E-MEXP-3603. Background correction and normalization of the raw data sets were performed using Robust MultiChip Analysis (RMA) implemented in "affy" R package [45].

\section{b). Clustering analysis}

For the clustering analysis the $\log _{2}$ of the relative gene expression levels in all the different transcriptional experiments considered was used. Hierarchical clustering of transcriptomic data was carried out with Expander software version $7.1[46,47]$ using the complete linkage option.

\section{Results}

Genes for asparagine metabolism in L. japonicus

Three genes encoding for asparagine synthetase were found in the L. japonicus genome by searching the available databases [48]: LjASN1 (accession number, Lj2g3v2291670.1), LjASN2 (accession number, Lj0g3v0295349.1) and LjASN3 (accession number, Lj0g3v0361789.1). According to phylogenetic analysis LjASN1 and LjASN2 belong to class I asparagine synthetases while LjASN3 belong to class II (Additional file 2: Figure S1). The genomic sequences of all these genes were analyzed. Thirteen, thirteen and fourteen exons were found in the genes encoding for LjASN1, LjASN2 and LjASN3 respectively (Additional file 3: Figure $\mathrm{S} 2$ ). The size of exons is similar comparing LjASN1 and LjASN2, but different for some particular exons in LjASN3.

In the case of asparaginase, three genes were identified: LjNSE1 (accession number, Lj5g3v0296030.1), LjNSE2 (accession number, Lj4g3v1736160.1), and LjNSE3 (accession 
number, Lj0g3v0303539.1) in the L. japonicus genome. Although the size of the genes was different, LjNSE1 and LjNSE2 genes had a similar structure composed of four exons, while five exons were found in the LjNSE3 gene (Additional file 3: Figure S2). According to the deduced amino acid sequence data LjNSE1 and LjNSE3 encode for $\mathrm{K}^{+}$- dependent asparaginases while LjNSE2 encodes for a $\mathrm{K}^{+}$- independent asparaginase, based on the previous analysis carried out for LjNSE1 and LjNSE2 enzymes [14].

Two genes encoding for serine-glyoxylate aminotransferase, LjSGAT1 (accession number, Lj6g3v0937010.1) and LjSGAT2 (accession number, Lj2g3v3058530.1), were also found in the $L$. japonicus genome. The gene structure and the number of exons were also similar between these two SGAT genes although both genes showed a different size.

\section{Transcriptional analysis of LjASN genes}

The levels of expression of the three genes encoding for ASN were determined by measuring by quantitative RTPCR the mRNA levels present in different organs of $L$. japonicus plants supplied with external nitrogen $\left(\mathrm{NH}_{4} \mathrm{NO}_{3}\right)$ or under purely symbiotic conditions. For comparative purposes, the measurements were carried out from samples taken either under light or dark conditions (Fig. 1). Under both $\mathrm{N}$ nutritions LjASN1 was highly expressed in mature leaves and roots, but its expression was insignificant in young leaves. The expression of LjASN1 was clearly up-regulated by light (about 2-fold) in mature leaves (Fig. 1A, B). In contrast, LjASN2 gene expression was negligible in leaves (Fig. 1C, D), while $L j A S N 3$ showed very low levels of expression compared to LjASN1 in this tissue (Fig. 1E, F). These results indicate that the expression of the LjASN1 gene must be

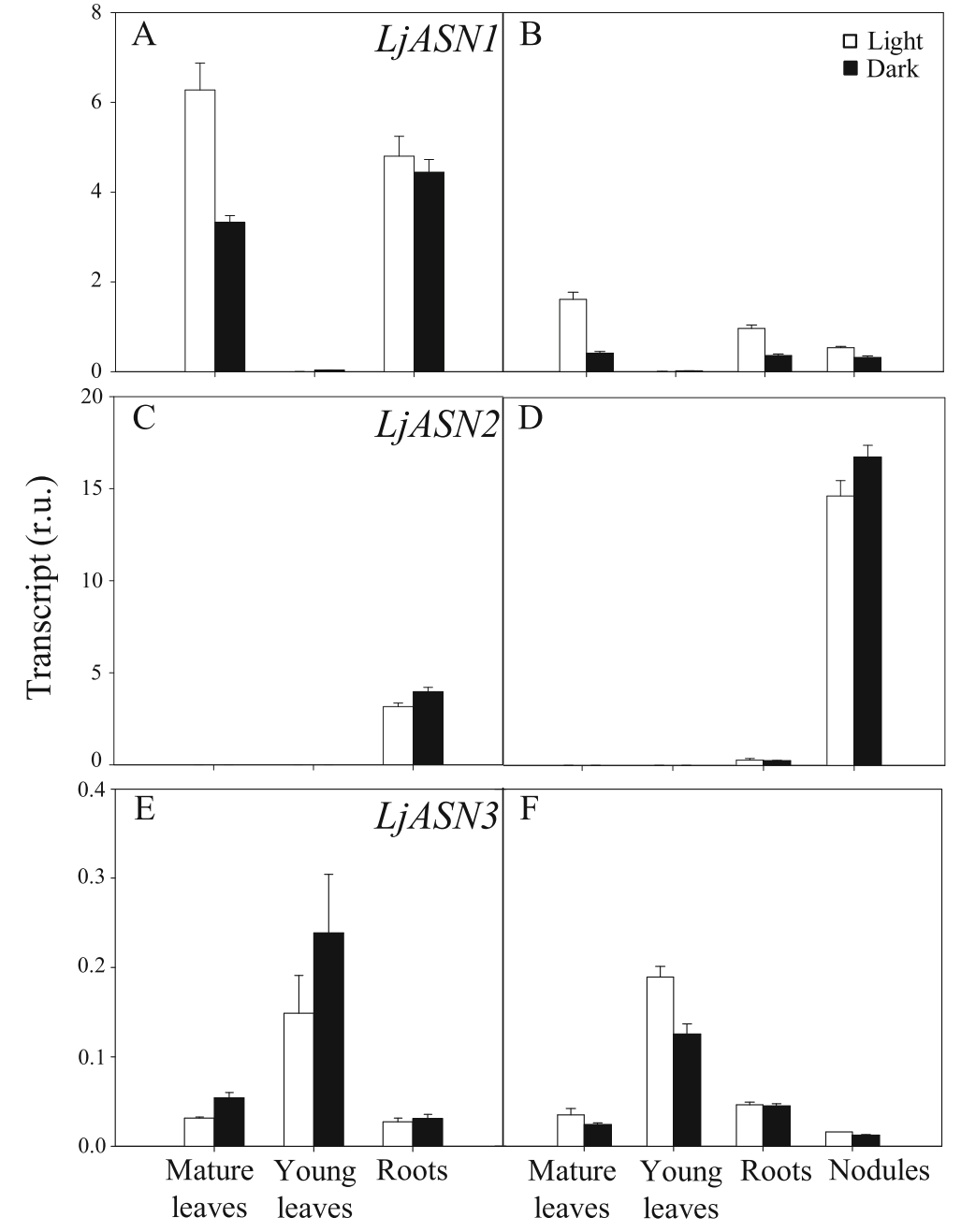

Fig. 1 qRT-PCR analysis of LjASN genes expression in different tissues of L. japonicus. Plants were grown for 6 weeks and irrigated with Hornum medium containing $\mathrm{NH}_{4} \mathrm{NO}_{3}(\mathbf{a}, \mathbf{c}, \mathbf{e})$, or inoculated with $M$. loti and irrigated with Hornum medium without nitrogen (b, $\mathbf{d}$, $\left.\mathbf{f}\right)$. The samples were harvested at $4 \mathrm{~h}$ after the beginning of the light or dark period for the quantification of transcripts. Transcript levels are reported as relative units (r.u.). Data are the mean \pm SE of three independent biological replicates 
crucially important for the biosynthesis of asparagine in mature leaves of $L$. japonicus. In young leaves of this plant, expression was only detectable for the LjASN3 gene, although its expression levels were quite low compared to the other $L j A S N$ genes in the other tissues examined.

All the three genes encoding for ASN were expressed in roots, particularly LjASN1 and LjASN2, although they were not generally affected by light (Fig. 1). This situation contrasts with the expression levels detected in nodules, where LjASN2 was the most highly expressed gene. Interestingly, very little expression of this particular LjASN2 gene was detected in the other organs examined from nodulated plants compared to nodules (Fig. 1D). These results indicate an important role of the LjASN2 gene for asparagine biosynthesis in nodules.

\section{Transcriptional analysis of LjNSE and LjSGAT genes}

The expression levels of LjNSE genes were also comparatively determined in nodulated plants and plants supplied with external N. LjNSE1 was the most highly expressed in all the plant tissues analysed with the exception of nodules (Fig. 2). Interestingly, the expression of this gene was stimulated by dark (2-fold) in mature leaves (Fig. 2A, B). The fact that this gene has more than 10 -fold higher expression than the other LjNSE genes in leaves, suggests that LjNSE1 is the main gene involved in asparagine catabolism in this organ. In contrast, the LjNSE2 transcript was detected in all plant tissues analyzed (Fig. 2C, D) and it was practically the only NSE gene expressed in nodules, thus suggesting a crucial role for this gene in asparagine degradation in this organ (Fig. 2D). It is worth noting that, the expression of LjNSE3 was significantly lower than that of LjNSE1 in
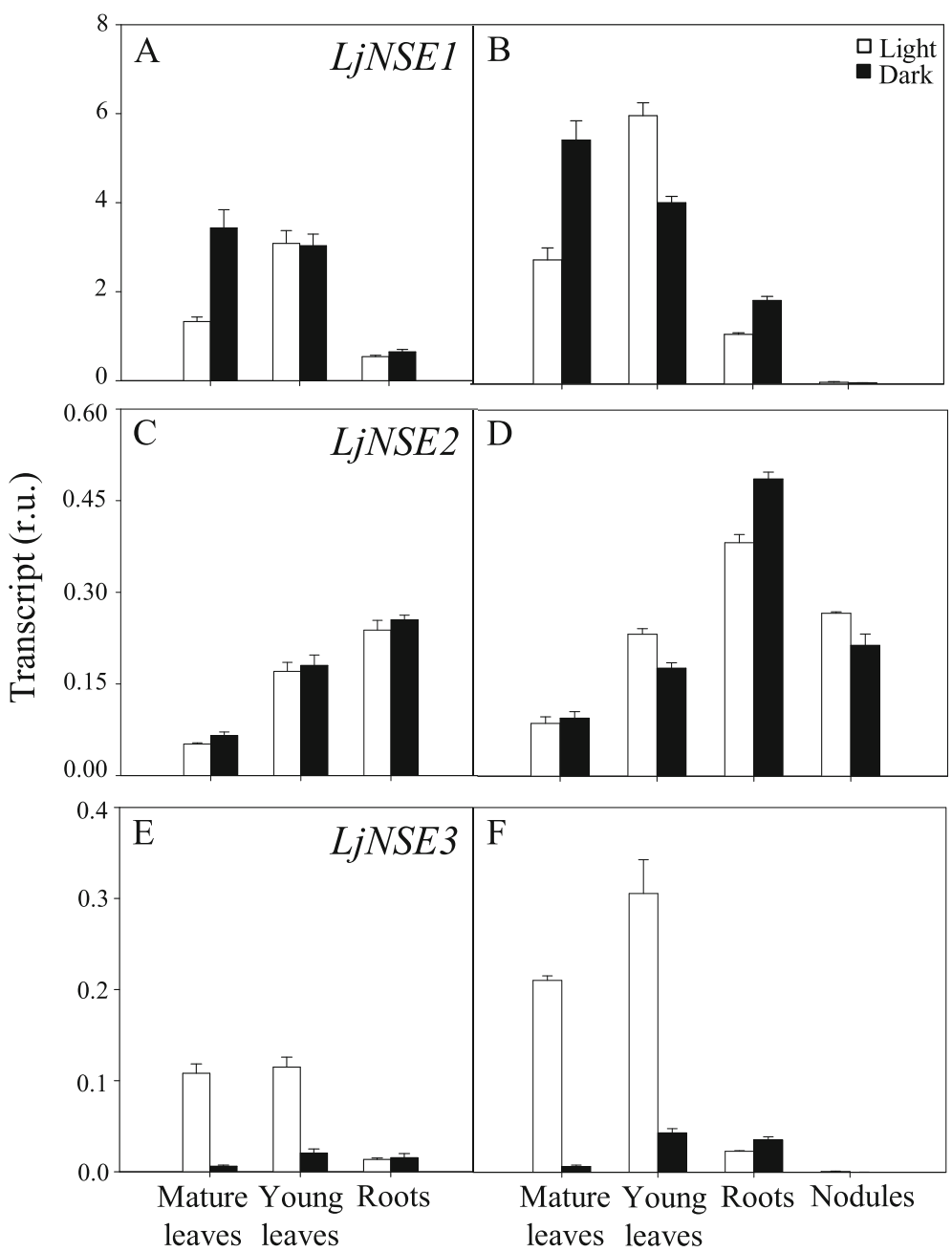

Fig. 2 qRT-PCR analysis of LjNSE genes expression in different tissues of L. japonicus. Plants were grown for 6 weeks and irrigated with Hornum medium containing $\mathrm{NH}_{4} \mathrm{NO}_{3}(\mathbf{a}, \mathbf{c}, \mathbf{e})$, or inoculated with $\mathrm{M}$. loti and irrigated with Hornum medium without nitrogen (b, $\mathbf{d}$, $\mathbf{f}$ ). The samples were harvested at $4 \mathrm{~h}$ after the beginning of the light or dark period for the quantification of transcripts. Transcript levels are reported as relative units (r.u.). Data are the mean \pm SE of three independent biological replicates 
leaves, roots and nodules (Fig. 2E, F), but a significant light-dependent expression was observed both in mature and young leaves (Fig. 2E, F).

With respect to the expression of the genes encoding for SGAT, LjSGAT2 was the most highly expressed gene in young and mature leaves. Its level of expression was 25-30 fold higher in comparison with LjSGAT1 (Fig. 3). However, the LjSGAT2 transcript was undetectable in roots and nodules (Fig. 3C, D). Interestingly, although the LjSGAT1 gene showed a much lower expression, this gene was found to be expressed in all the organs analysed: leaves, roots and nodules, and it was highly lightinduced in leaves (Fig. 3A, B).

\section{A mutant deficient in NSE1 asparaginase shows a dramatic diminution of the transcript levels of LjSGAT genes}

The possible changes in expression of asparagine metabolism genes were also examined in a TILLING mutant available, called Ljnse1-4, which was specifically affected in LjNSE1 asparaginase and accumulated high levels of asparagine [15]. In the present paper no changes were detected in the expression levels of asparagine synthetase and asparaginase genes between WT and Ljnse1-4 mutant plants under all the conditions considered. However, a dramatic reduction of the transcript levels of LjSGAT1 and LjSGAT2 was detected in leaves of the
Ljnse1-4 mutant compared to the WT plants (Fig. 4) in spite of the fact that no changes in expression levels of LjSGAT genes were observed in roots and nodules (not shown). It can be concluded that the deficiency of LjNSE1 has a very important influence in the expression of the genes encoding for SGAT. Considering that SGAT has been previously associated to the $\mathrm{N}$ photorespiratory cycle in other plant species [16, and references therein], the results obtained could be taken as an indication of the existence of a close relationship between the expression of genes for asparagine metabolism and photorespiratory genes in L. japonicus.

\section{Changes in the transcription of genes for asparagine metabolism in a Ljgln2 photorespiratory mutant}

The previous results gave rise to the study of the possible connection between asparagine metabolism and the photorespiratory process in L. japonicus. For this purpose, the plants were grown under a $\mathrm{CO}_{2}$-enriched atmosphere $(0.7 \% \mathrm{v} / \mathrm{v})$ where photorespiration is suppressed and transferred to a normal air atmosphere (about $0.04 \% \mathrm{CO}_{2}$ ) where photorespiration is active. A $L$. japonicus photorespiratory mutant, $\operatorname{Ljg} \ln 2-2$, deficient in the plastidic isoform of glutamine synthetase $\left(\mathrm{GS}_{2}\right)$ that was previously isolated and characterized at the molecular level in our laboratory [28, 32] was also included in our study to determine the influence of the impairment

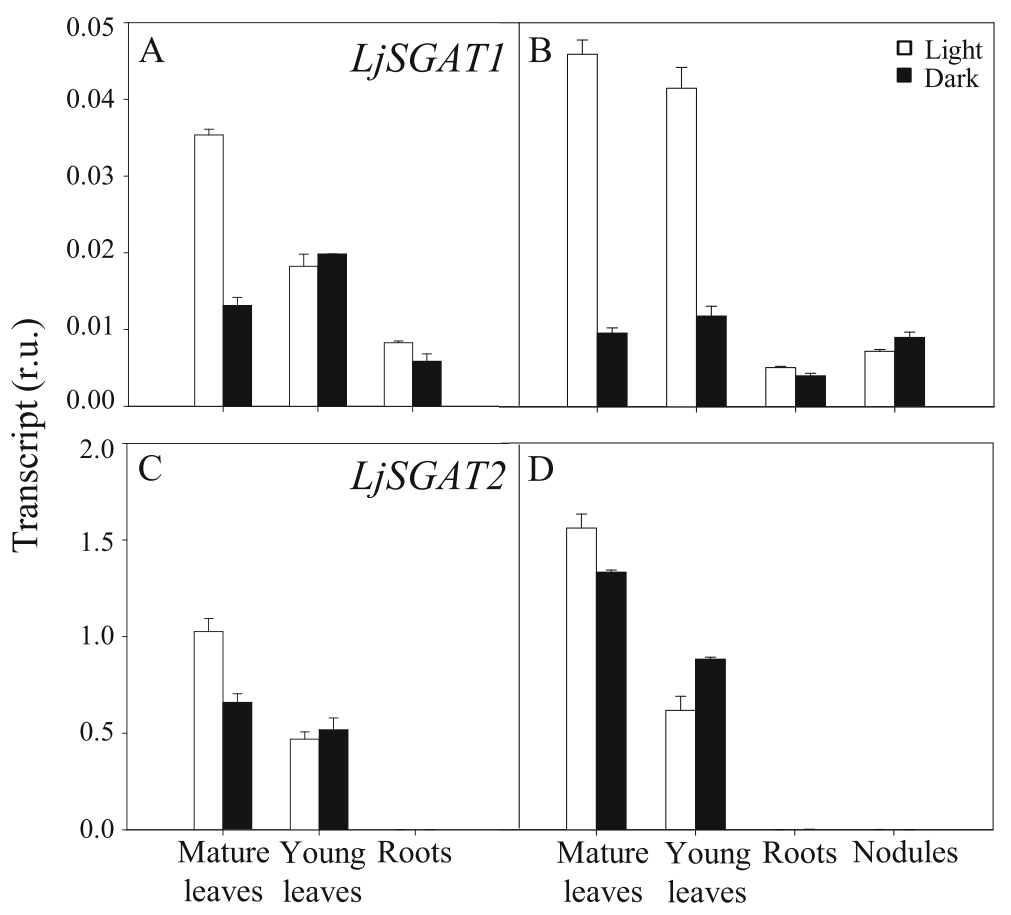

Fig. 3 qRT-PCR analysis of LjSGAT genes expression in different tissues of L. japonicus. Plants were grown for 6 weeks and irrigated with Hornum medium (a, c), containing $\mathrm{NH}_{4} \mathrm{NO}_{3}$ or inoculated with $\mathrm{M}$. loti and irrigated with Hornum medium without nitrogen (b, d). The samples were harvested at $4 \mathrm{~h}$ after the beginning of the light or dark period for the quantification of transcripts. Transcript levels are reported as relative units (r.u.). Data are the mean \pm SE of three independent biological replicates 

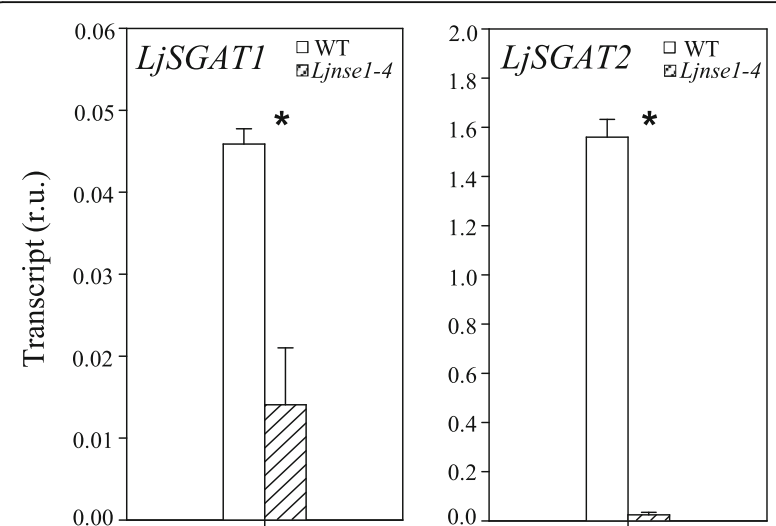

Fig. 4 qRT-PCR analysis of LjSGAT genes expression in leaves of WT and Ljnse1-4 mutant plants. L. japonicus plants were grown for 6 weeks under normal air conditions. Leaves were harvested at $4 \mathrm{~h}$ after the beginning of the light period. Transcript levels are reported as relative units (r.u.). Data are the mean \pm SE of three independent biological replicates. * indicates significant differences between WT and Ljnse $1-4$ mutant plants as determined by Student's test $(P<0.05)$

of the photorespiratory cycle on asparagine metabolism. It has been previously shown that this plant accumulated significant amounts of ammonium and asparagine when transferred from high $\mathrm{CO}_{2}$ to air as a result of $\mathrm{GS}_{2}$ deficiency [39].

The expression levels of genes involved in asparagine metabolism in the WT and mutant plants were determined at different time points after the transfer from high $\mathrm{CO}_{2}$ (suppressed photorespiration) atmosphere to normal air (active photorespiration) conditions. Considering that photorespiration takes place exclusively in leaves, the analyses were only carried out in this organ. The expression levels of LjASN1 were similar in high $\mathrm{CO}_{2}$ and air conditions in WT plants (Fig. 5). However, LjASN1 expression levels diminished significantly in the Ljgln2-2 mutant after the transfer to normal air (Fig. 5). On the other hand, LjASN2 showed a slight diminution in WT plants after the transfer to normal air, recovering after 10 days under photorespiratory conditions. By contrast, the levels of expression of the LjASN2 gene showed a remarkable increase in Ljgln2-2 mutant plants after the transfer to active photorespiratory conditions (Fig. 5). LjASN3 gene expression diminished both in WT and $\operatorname{Lg} \ln 2-2$ plants after the transfer to air, and this diminution was more marked in the mutant genotype.

Several differences were also detected between WT and $L j g \ln 2-2$ photorespiratory mutant plants in regards to the expression of asparaginase genes. LjNSE1 expression decreased significantly after 10 days of the transfer to air in the mutant but not in the WT (Fig. 6). LjNSE2 expression levels decreased strongly both in WT and Ljgln2-2 mutant plants. LjNSE3 expression levels gradually decreased in the WT after the transfer to normal

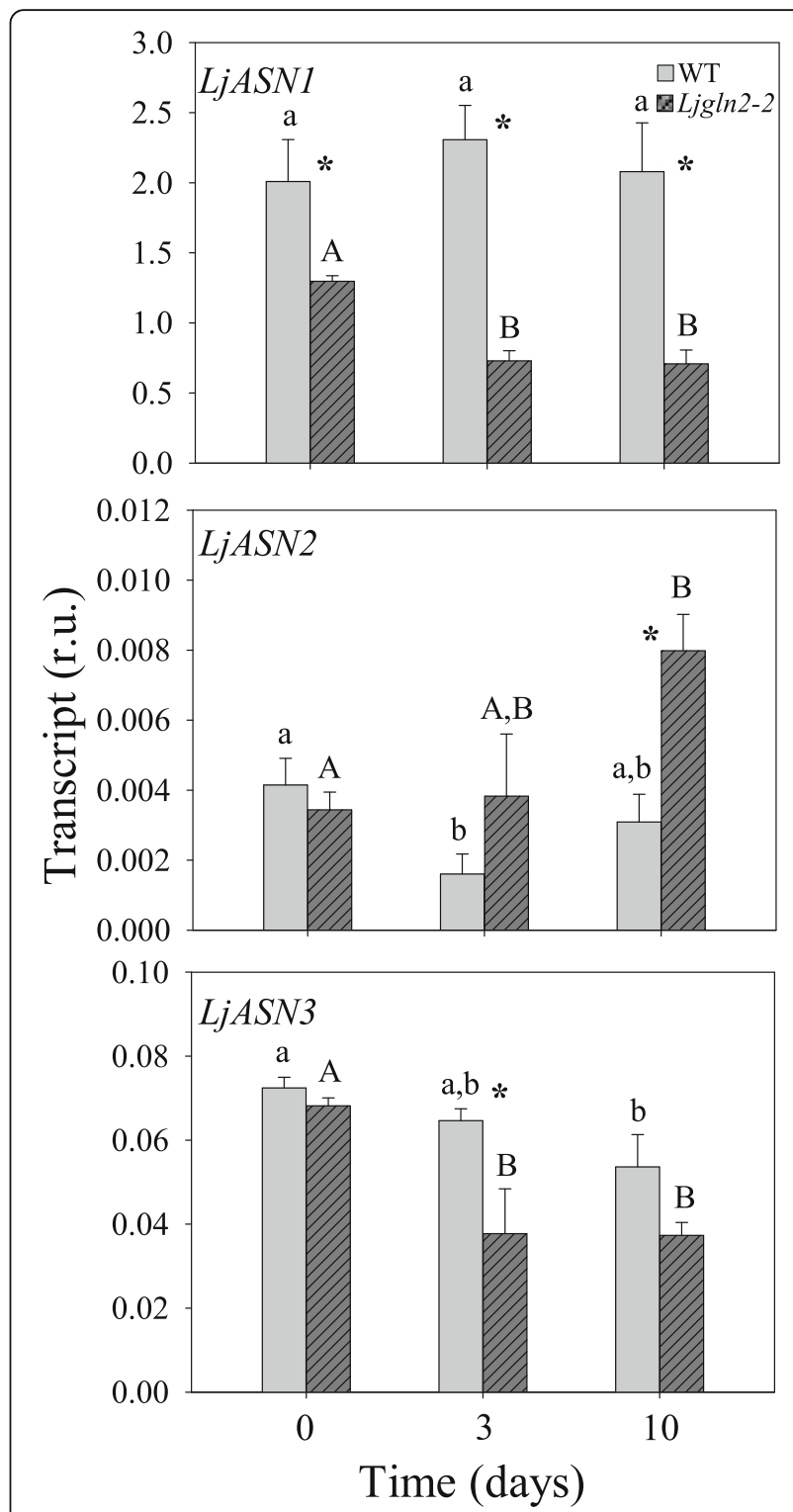

Fig. 5 qRT-PCR analysis of LjASN genes expression in leaves of WT and Ljgln2-2 mutant plants. L. japonicus plants grown for 35 days in high $\mathrm{CO}_{2}$ (time zero) were transferred to normal $\mathrm{CO}_{2}$ conditions for the indicated periods of time. Leaves were harvested at the indicated time points. Transcript levels are reported as relative units (r.u.). Data are the mean \pm SE of three independent biological replicates. * indicates significant differences between WT and LjgIn2-2 mutant plants; $\mathrm{a}, \mathrm{b}$ indicate significant differences between $\mathrm{CO}_{2}$ and air conditions at the indicated time points for WT plants; A, B indicate significant differences between $\mathrm{CO}_{2}$ and air conditions at the indicated time points for $\mathrm{Ljg} / \mathrm{n} 2-$ 2 plants as determined by Student's test $(P<0.05)$

air and were also significantly diminished in the mutant, but only at day 3 (Fig. 6).

The expression levels of $L j S G A T$ genes were also determined in both genotypes. No major changes were observed in the expression levels of LjSGAT1 either in WT or mutant plants. However, the level of expression of the 


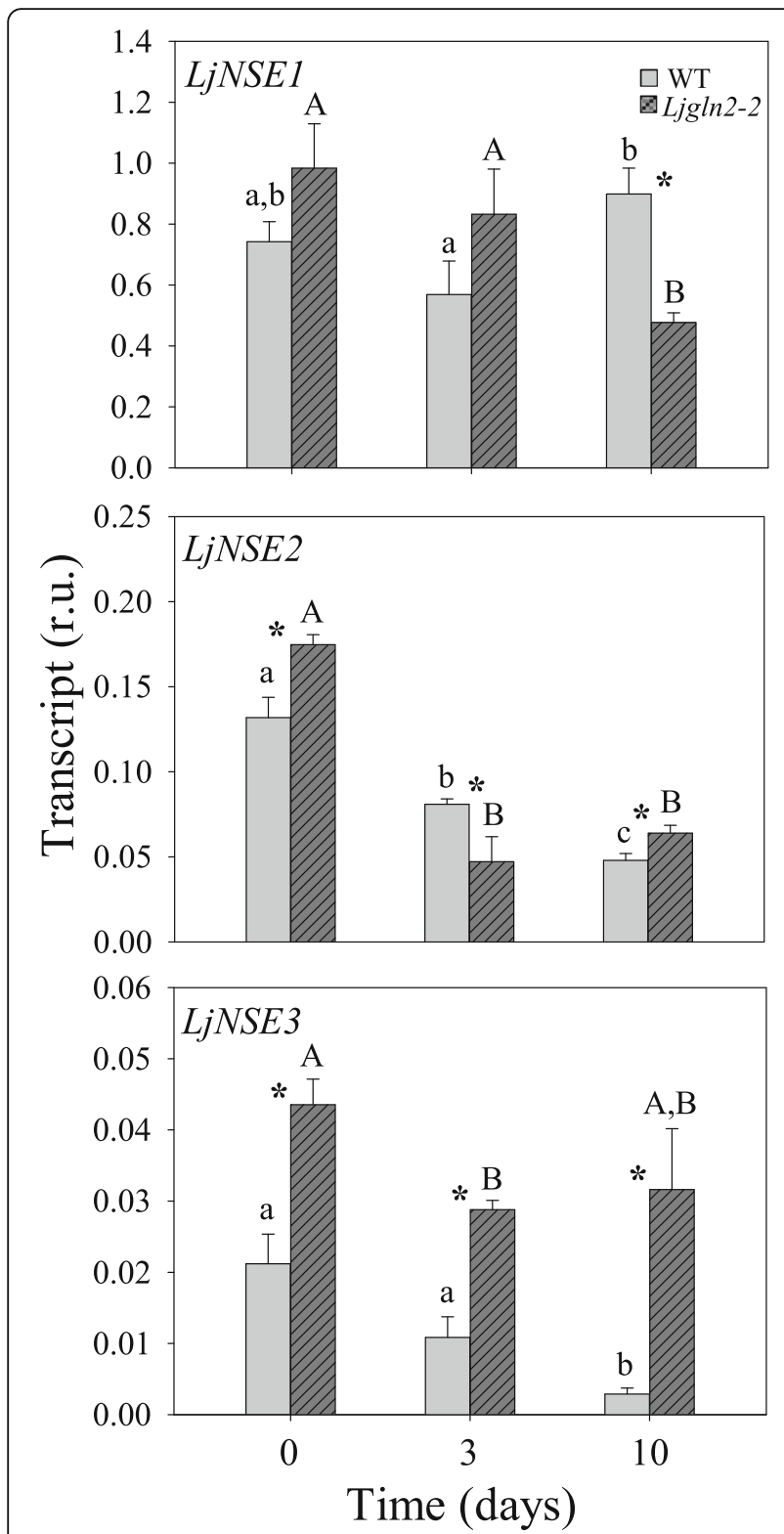

Fig. 6 qRT-PCR analysis of LjNSE genes expression in leaves of WT and Ljgln2-2 mutant plants. L. japonicus plants grown for 35 days in high $\mathrm{CO}_{2}$ (time zero) were transferred to normal $\mathrm{CO}_{2}$ conditions for the indicated periods of time. Leaves were harvested at the indicated time points. Transcript levels are reported as relative units (r.u.). Data are the mean \pm SE of three independent biological replicates. * indicates significant differences between WT and LjgIn2-2 mutant plants; $a, b, c$ : indicate significant differences between $\mathrm{CO}_{2}$ and air conditions at the indicated time points for WT plants; A, B: indicate significant differences between $\mathrm{CO}_{2}$ and air conditions at the indicated time points for $\mathrm{Ljg} / \mathrm{n} 2-$ 2 plants as determined by Student's test $(P<0.05)$

LjSGAT2 gene was 15-fold higher than the expression of LjSGAT1 and decreased substantially after 3 days of the transfer of plants from high $\mathrm{CO}_{2}$ to air conditions, particularly in the mutant plants, followed by some recovery after 10 days under air atmosphere (Fig. 7).

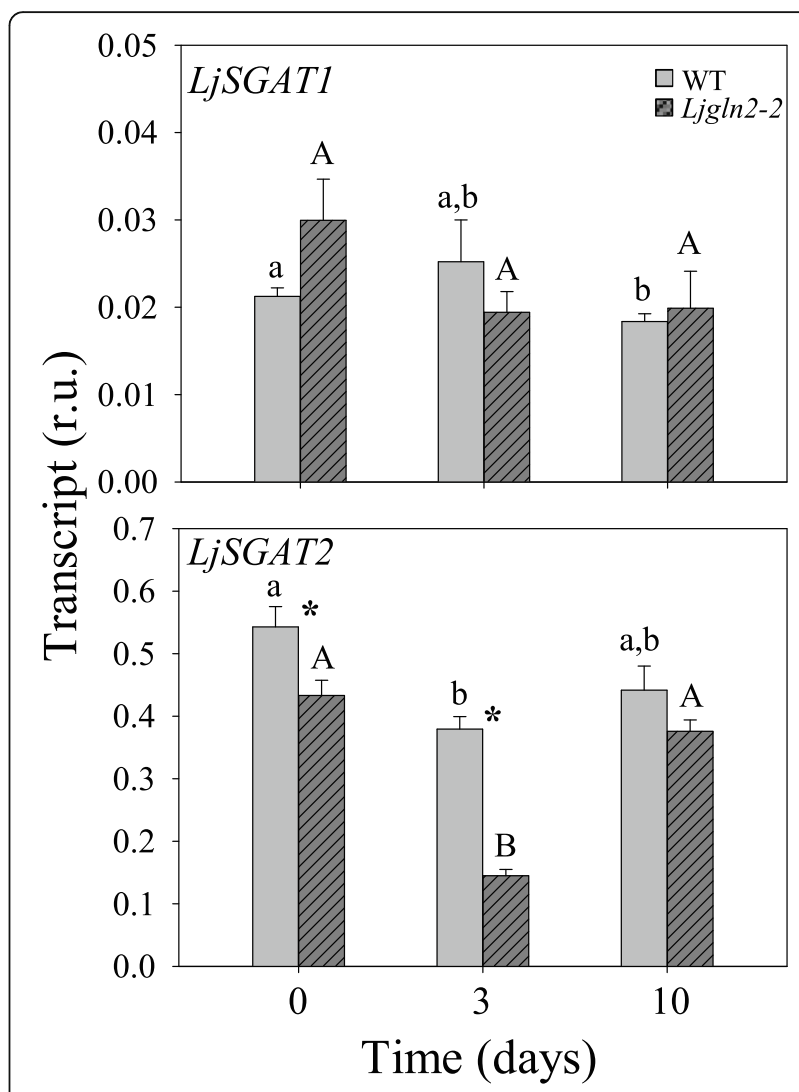

Fig. 7 qRT-PCR analysis of LjSGAT genes expression in leaves of WT and Ljgln2-2 mutant plants. L. japonicus plants grown for 35 days in high $\mathrm{CO}_{2}$ (time zero) were transferred to normal $\mathrm{CO}_{2}$ conditions for the indicated periods of time. Leaves were harvested at the indicated time points. Transcript levels are reported as relative units (r.u.). Data are the mean \pm SE of three independent biological replicates. ${ }^{*}$ indicates significant differences between WT and LjgIn2-2 mutant plants; a, b: indicate significant differences between $\mathrm{CO}_{2}$ and air conditions at the indicated time points for WT plants; A, B: indicate significant differences between $\mathrm{CO}_{2}$ and air conditions at the indicated time points for $\mathrm{Ljg} / \mathrm{h} 2-$ 2 plants as determined by Student's test $(P<0.05)$

Noteworthy, the trend of transcriptional regulation observed for LjASN1 and LjSGAT2 in the Ljgln2-2 mutant was found to be quite similar to the one reported in a previous work for most of the photorespiratory genes [39]. A sudden drop in transcript levels was observed upon the transfer from high $\mathrm{CO}_{2}$ (suppressed photorespiration) to normal air (active photorespiration) atmosphere, followed by a recovery in some cases of the transcript levels. For this reason, a hierarchical clustering was carried out based on the transcript levels determined at different times of the transfer to air for the different asparagine metabolism genes that were analyzed in the present work, together with those of all known photorespiratory genes and some other nitrogen metabolism genes from L. japonicus. Two main clusters of genes were obtained: one that contained most genes for $\mathrm{N}$ metabolism (upper cluster in Fig. 8) and another one 


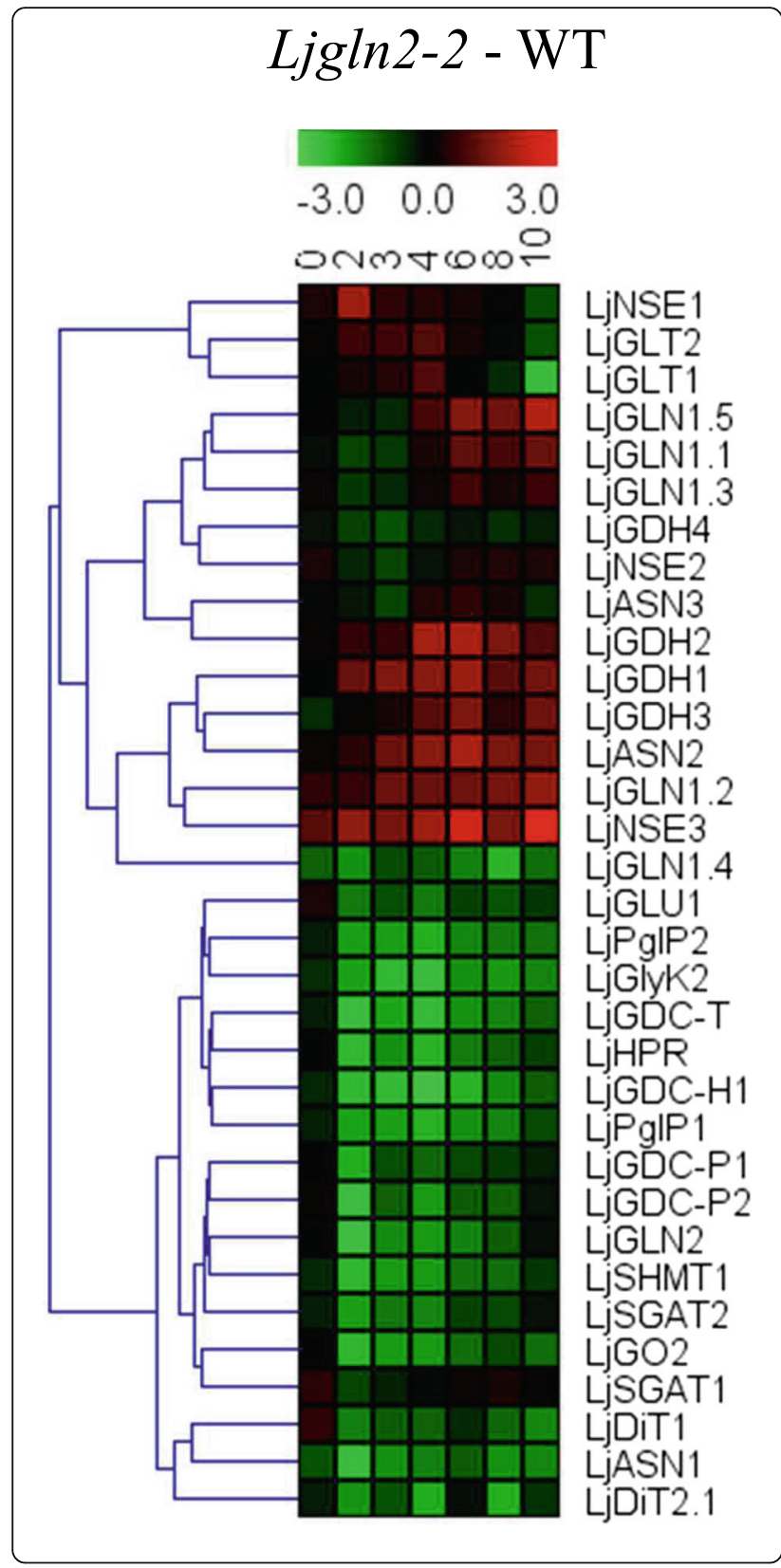

Fig. 8 Hierarchical clustering analysis of quantitative RT-PCR data for photorespiratory and nitrogen metabolism genes. Transcript levels were determined at the indicated time periods after the transfer of WT and Ljg/n2-2 mutant plants from $\mathrm{CO}_{2}$-enriched (time zero) to normal $\mathrm{CO}_{2}$ conditions. Relative transcript levels of WT plants under $\mathrm{CO}_{2}$-enriched atmosphere were taken as 1. Data are presented as the log2 of the difference of transcript levels between WT and Ljgln2-2. The genes considered and their corresponding accession numbers according to the Kazusa database are: asparagine synthetase (LjASN1, Lj2g3V2291670.1; LjASN2, Lj0g3v0295349.1; LjASN3,

Lj0g3v0361789.1); asparaginase (LjNSE1, Lj5g3v0296030.1; LjNSE2, Lj4g3v1736160.1; LjNSE3, Lj0g3V0303539.1); serine hydroxymethyltransferase (LjSHMT1, Lj2g3v1467880.1); ferredoxin-dependent GOGAT (LjGLU1, Lj1g3v4154900.1); NADH-GOGAT (LjGLT1, LjSGA_035611.1*; LjGLT2, LjSGA_037992.1*); serine:glyoxylate aminotransferase (LjSGAT1, Lj6g3v0937010.1; LjSGAT2, Lj2g3v3058530.1); plastidic glutamine synthetase (LjGLN2, Lj6g3v1887800.1); cytosolic glutamine synthetase (LjGLN1.1, Lj2g3v0658180.1; LjGLN1.2, Lj6g3v0410490.1; LjGLN1.3, Lj0g3v0335159.1; LjGLN1.4, LjSGA_058827.1*; LjGLN1.5, LjSGA_019428.1*); glutamate dehydrogenase (LjGDH1, Lj1g3v3975110.1; LjGDH2, Lj4g3v1212370.1; LjGDH3, Lj2g3v1988990.1; LjGDH4, Lj0g3v0102829.1); hydroxypyruvate reductase (LjHPR, Lj5g3v2242500.1); glycine decarboxylase (LjGDC-H1, Lj4g3v0654460.1; LjGDC-P1, chr5.CM0019.20.r2.m*; LjGDC-P2, chr5.LjT34K16.170.r2.m*; LjGDC-T, Lj6g3v1849480.1); glycerate kinase (LjGlyK2, Lj3g3v2247080.1); glycolate oxidase (LjGO2, Lj3g3v1048900.2); plastidic dicarboxylate transporter (LjDiT1, chr5.CM0089.610.r2.d*; LjDiT2.1, Lj6g3v2204740.1) and phosphoglycolate phosphatase (LjPgIP1, Lj1g3v2842370.1; LjPgIP2, Lj6g3v1708420.1). The gene accession numbers are reported according to the version 3.0 of the L. japonicus genome in the Kazusa database, except in the cases indicated with an asterisk where the version 2.5 of the genome was used

(lower one in Fig. 8) that contained most photorespiratory genes. Very interestingly, LjASN1, LjSGAT1 and LjSGAT2 genes clustered together with the photorespiratory genes. On the other hand, LjASN2, LjASN3, LjNSE1, LjNSE2 and LjNSE3 clustered together with the genes encoding for other enzymes of nitrogen metabolism (Fig. 8). Therefore we conclude that among all asparagine metabolism genes analyzed in the present paper, $L j A S N 1$ and both LjSGAT1 and LjSGAT2 genes are those most likely connected with photorespiratory metabolism in $L$. japonicus plants.

\section{Transcriptomic analysis of asparagine metabolism genes}

The pattern of expression of the different genes involved in asparagine metabolism were comparatively examined together with other genes for photorespiratory metabolism and nitrogen metabolism, using for this purpose an integration of all the transcriptomic data available for $L$. japonicus from 84 different physiological conditions and/or tissues (Additional file 4: Table S2). Figure 9 shows a clustering analysis carried out from all these transcriptomic data. A close link was obtained between LjASN1 gene expression and the expression of most of the photorespiratory metabolism related genes. This was also the case for LjSGAT1 and LjSGAT2 genes, which were associated too to LjASN1 and other photorespiratory genes. 


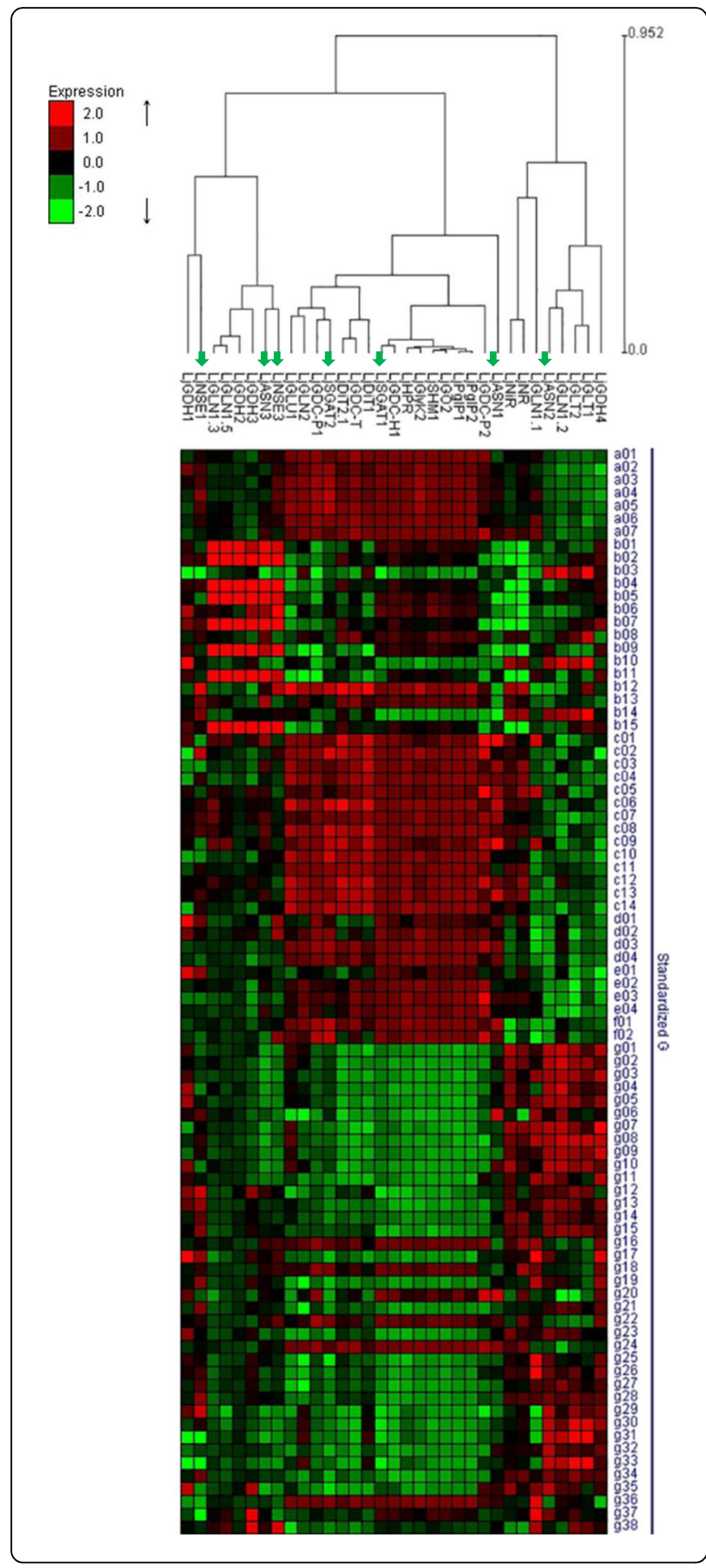

Fig. 9 Clustering analysis of transcript levels of asparagine metabolism genes and photorespiratory and $\mathrm{N}$ metabolism genes. The clustering analysis was carried out with the Expander software using complete linkage. The mean of the expression level of each gene in all the samples analyzed was calculated and transformed in a $\log _{2}$ before clustering analysis. In the color panels, each vertical line represents a single gene, and the color of the line indicates the expression level (in a log scale) of the gene relative to a specific sample: high expression in red, low expression in green. The photorespiratory genes and genes of nitrogen metabolism present in the clustering image are: asparagine synthetase (LJASN1, LjASN2 and LJASN3); asparaginase (LJNSE1 and LjNSE3); nitrate reductase (LjNR, Lj0g3v0006719.1); nitrite reductase (LjNiR, Lj4g3v0588830.1); serine hydroxymethyltransferase (LSSHM1); ferredoxin-dependent glutamate synthase (LjGLU1); NADH-dependent glutamate synthase (LjGLT1 and LjGLT2); serine:glyoxylate aminotransferase (LjSGAT1 and LjSGAT2); plastidic glutamine synthetase (LjGLN2); cytosolic glutamine synthetase (LjGLN1.1, LjGLN1.2, LjGLN1.3 and LjGLN1.5) glutamate dehydrogenase ( $L j G D H 1$, $L j G D H 2$, $L j G D H 3$ and $L j G D H 4)$; hydroxypyruvate reductase (LjHPR); glycine decarboxylase (LjGDC-H1, LjGDC-P1, LjGDC-P2 and $L j G D C-T)$; glycerate kinase ( $L j G / y K 2)$; glycolate oxidase ( $L j G O 2)$; plastidic dicarboxylate transporter (LjDiT1 and LjDiT2.1) and phosphoglycolate phosphatase (LjPg/P1 and LjPg/P2). The accession numbers of the genes mentioned previously (Fig. 8) can be found in the corresponding figure legend

Therefore, the transcriptomic analysis carried out confirms again the results previously shown in this paper that establish a clear link between LjASN1 and LjSGAT1 and LjSGAT2 genes with photorespiratory metabolism in $L$. japonicus. In contrast, the pattern of expression of the LjASN2 gene was more closely associated with a completely different set of genes, mainly encoding for proteins involved in nitrogen assimilation such as nitrate reductase (NR), nitrite reductase (NiR), cytosolic glutamine synthetase (GS1) or NADH-dependent glutamate synthase (NADH-GOGAT). Moreover, it was also shown that the LjASN2 gene was more highly expressed in roots and nodules than in leaves, as deduced from the corresponding transcriptomic data available from these organs. Consequently, the results obtained from the analysis of the expression of the LjASN2 gene from an ample set of transcriptomic data are in agreement with the previous results shown in the present paper.

\section{Discussion}

The results obtained in the present paper indicate the existence of a differential expression of various genes involved either in asparagine biosynthesis or degradation in this plant. Figure 10 summarizes in a schematic form the main results obtained in regard to the different pattern of expression found for asparagine synthetase, asparaginase, and SGAT genes in different tissues such as young or mature leaves, nodules and roots. The results obtained point out an important difference in the levels of expression of $A S N$ genes among mature leaves and young leaves of $L$. japonicus plants. A much higher 


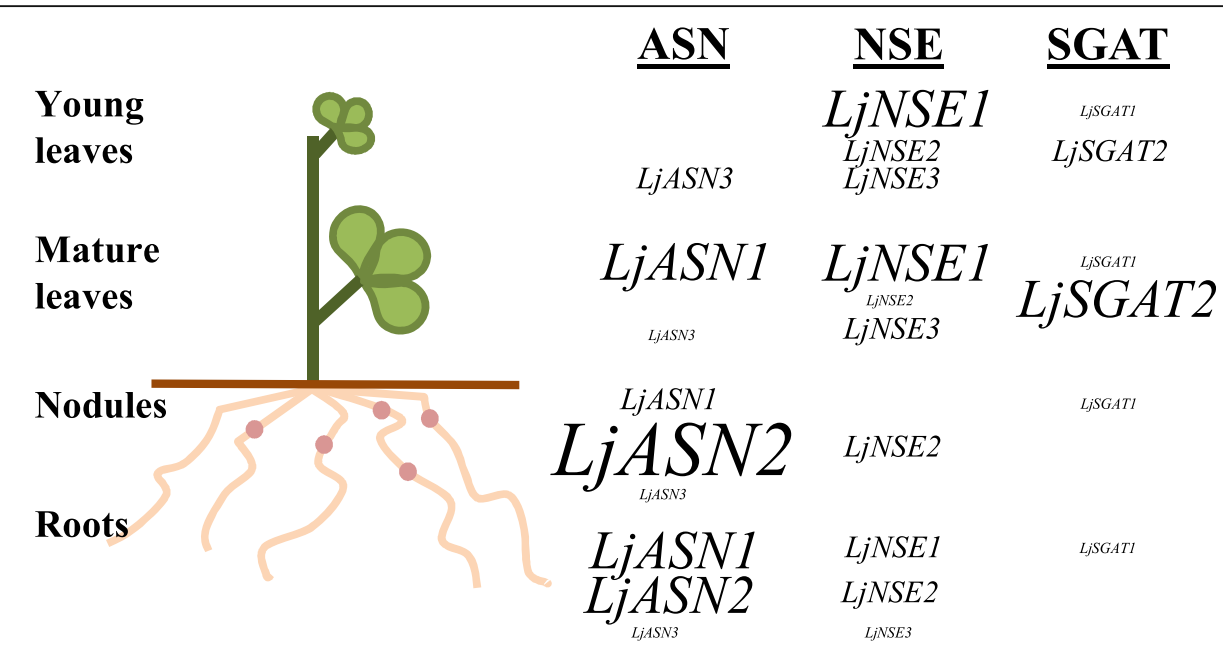

Fig. 10 Differential expression of the different genes encoding for LjASN, LjNSE and LjSGAT in L. japonicus. The size of the lettering reflects the relative abundance of the different genes analyzed in the paper. Genes mentioned in this figure are the key genes for the following biochemical pathways: LjASN, asparagine biosynthesis; LjNSE, asparagine degradation; LjSGAT, photorespiration/transamination

level of expression was obtained for the LjASN1 gene in mature leaves, compared to the young ones. This result would be consistent with a major role of the mature leaves as a source tissue for exporting asparagine to the young growing leaves of the plant, in accordance with the crucial involvement of asparagine as a $\mathrm{N}$ translocator in L. japonicus [2]. The high levels of LjNSE1 asparaginase gene expression in young leaves indicated a high potential for asparagine utilization in this type of tissue of the plants. The fact that LjNSE1 was the most highly expressed gene in all the plant tissues analysed, with the exception of nodules, suggested that the LjNSE1 gene (which encodes a $\mathrm{K}^{+}$-dependent asparaginase) must be the most crucial gene for asparagine catabolism in leaves. Credali et al. [15] proved the crucial relevance of LjNSE1 for plant growth and seed production of $L$. japonicus.

On the other hand, the fact that the expression of the LjASN2 gene is restricted to the non-photosynthetic tissues examined, such as roots and nodules, suggests a crucial involvement of this gene for the particular metabolism of these organs. The very high levels of transcription of the LjASN2 gene found in nodules seem particularly interesting since asparagine is one of the amino acids involved in the nitrogen export from the nodules. Prell and Poole [49] described that the amino acid cycling process is crucially relevant in symbiosis suggesting that, in addition to the exchange of carbon compounds and nitrogen fixed, plants and bacteroids also exchange amino acids. It is quite likely that the LjASN2 gene may be crucial for the synthesis of asparagine in nodules, which is subsequently transported to the plant. In addition, the fact that only one particular asparaginase gene (LjNSE2) was expressed in nodules suggests an interesting role of this gene in nodule function.
The fact that LjASN1 gene expression was shown to be light stimulated in L. japonicus (Fig. 1) is very interesting since class-I asparagine synthetases are usually up-regulated in the dark in response to a low carbon:nitrogen ratio. However, our results are in agreement with the previous data obtained by Waterhouse et al. [2] in the same plant, although in our case quantitative data of gene expression is provided. The light induction of LjASN1 may be related with the fact that asparagine constitutes the major $\mathrm{N}$-transport compound to undertake $\mathrm{N}$-assimilation in L. japonicus, and nitrate reduction would be maximal in the light. In addition, in the present paper several lines of evidence establish clear links between LjASN1 and photorespiration in L. japonicus, which could be taken as another possible explanation of the light inducibility of LjASN1 gene. In fact, gene expression analysis (Figs. 8 and 9) indicated that the transcriptional regulation of $L j A S N 1$, together with those of LjSGAT1 and LjSGAT2, were very similar to photorespiratory genes and quite different to other examined genes. Moreover, a further link between asparagine metabolism and photorespiration would come from the dramatic decrease in the expression of both LjSGAT1 and LjSGAT2 genes that occurs in the Ljnse1-4 asparaginase deficient mutant from L. japonicus (Fig. 4). This mutant was shown to have much higher levels of asparagine [15]. Changes in SGAT expression in this asparaginase mutant could be related to the fact that asparagine plays an important role on the regulation of $\mathrm{N}$ flux in the $\mathrm{N}$-organic pool [50] and that Modde et al. [51] have recently concluded that SGAT needs to be dynamically adjusted to ensure a variable flux through the photorespiratory pathway.

If there is any possible role of $L j A S N 2$ in relation to photorespiration remains intriguing. The pattern of 
expression of $L j A S N 2$ gene seems to be completely different to those of LjASN1 and LjSGAT genes (Figs. 1, 8 and 9), thus not showing a clear link with photorespiration. Furthermore, the work carried out by Gaufichon et al. [9] with ASN2 mutants argued that this gene does not contribute to photorespiratory N cycle. However, Fig. 5 shows that $L j A S N 2$ is increased on the transfer from high- $\mathrm{CO}_{2}$ (suppressed photorespiration) to normal air (active photorespiration) conditions in the photorespiratory mutant Ljgln2-2 lacking plastidic GS. Pérez-Delgado et al. [52] have shown that there is a strong increase in LjASN2 gene expression, concomitant with the increase in photorespiratory ammonium produced as a result of the lack of plastidic GS. This increase in LjASN2 gene expression takes place in parallel with an increase in asparagine levels in the same tissues that occurs simultaneously with an increase in glutamine due to the induction of cytosolic GS1 in the mutant [52]. It was concluded that this increase in LjASN2 and asparagine forms part of the different responses of the plant to detoxify the high levels of photorespiratory ammonium produced by the absence of plastidic GS. A good correlation between ASN2 gene expression and asparagine content was also found in Arabidopsis. The asparagine contents in ASN2- overexpressing and underexpressing plants were increased and decreased, respectively, when they were grown under normal light and nutrient conditions, suggesting that ASN2 functions as a regulator of asparagine biosynthesis and metabolism and that it mediates the effective use of nitrogen under ammonium sufficient conditions [53]. Other types of evidence have proposed that $A S N 2$ is induced by ammonium treatments and by stress treatments that cause ammonium accumulation, suggesting a physiological role of ASN2 related, directly or indirectly, to the reassimilation of the nitrogen remobilized under stress conditions [7]. A possible relationship between the level of ASN2 gene expression and the level of ammonium loss via the photorespiratory pathway has been also described [7]. Several authors have reported the possibility of ammonium dependent synthesis of asparagine in plants [4].

\section{Conclusions}

The results obtained in this paper indicate the existence of a differential expression of asparagine metabolic genes in L. japonicus and point out the crucial relevance of particular genes in different organs. Moreover, the data presented establish clear links between asparagine and photorespiratory metabolic genes in this plant.

\section{Additional files}

Additional file 1: Table S1. Sequences of primers used in $\mathrm{QRT} T-\mathrm{PCR}$ experiments. (DOC $35 \mathrm{~kb}$ )
Additional file 2: Figure. S1. Phylogenetic tree of asparagine synthetases. A dendrogram of asparagine synthetase sequences was generated by PILEUP as previously described [5] including LjASN1, LjASN2 and LjASN3 from L. japonicus. Class-I and class-II phylogenetic clades are shown. (TIFF $626 \mathrm{~kb}$ )

Additional file 3: Figure. S2. Structures of the LJASN, LJNSE and LJSGAT genes from L. japonicus. Exons are represented as boxes. (TIFF $134 \mathrm{~kb}$ )

Additional file 4: Table S2. List and description of the L. japonicus transcriptomic data analyzed in the present work. All transcriptomic data are available online in the Arrayexpress database. (XLSX 14 kb)

\section{Abbreviations}

ASN: asparagine synthetaseATPadenosine triphosphateGS1cytosolic glutamine synthetaseGS2plastidic glutamine synthetaseLjGPIL. japonicus glycosylphosphatidyl inositol-anchored proteinLjHAR1L. japonicus hypernodulation aberrant root formationLjPp2AL. japonicus protein phosphatase 2ALjUbc10L. japonicus ubiquitin carrier protein 10LjUbqL. japonicus polyubiquitin 4NADH-GOGATNADH-dependent glutamate synthaseNiRnitrite reductaseNRnitrate reductaseNSEasparaginaseqRTPCRquantitative real-time polymerase chain reactionSGATserine-glyoxylate aminotransferaseTILLINGTargeted Induced Local Lesions IN GenomesWTwild type

\section{Acknowledgments}

We thank the biology facilities of the Centro de Investigación Tecnológica e Innovación (CITIUS) of the University of Seville for quantitative RT-PCR measurements, and M.J. Cubas and A. Gómez for technical and secretarial assistance.

\section{Funding}

The work was supported by Junta de Andalucía (research project P10-CVI6368) and FEDER-Ministerio de Economía y Competitividad (Spain) (project AGL 2014-54413-R). C.M.P. acknowledges a fellowship given by the $V$ Plan Propio from University of Seville (Spain). The funding agencies were not involved in the design of the study, collection, analysis, and interpretation of data and in writing the manuscript.

\section{Availability of data and materials}

The data supporting the conclusions of this article are included within the article and its additional files. The microarray data analyzed in this article are publically available in the ArrayExpress database (https://www.ebi.ac.uk/ arrayexpress/). The code numbers of the experiments are: E-MEXP-1204, ETABM-715, E-MEXP-2344, E-MEXP-2690, E-MEXP-1726, E-MEXP-3710 and EMEXP-3603.

\section{Authors' contributions}

All the authors jointly conceived and designed the experiments and carried out the data analysis. MG-C, CMP-D and AC conducted the experiments. MGC, JMV, MB and AJM wrote the manuscript. All the authors reviewed and approved the final manuscript.

\section{Ethics approval and consent to participate}

L. japonicus (Regel) Larsen cv. Gifu was initially obtained from Prof. Jens Stougaard (University of Aarhus, Denmark) and then self-propagated at the University of Seville. The L. japonicus Ljgln2-2 mutant used in this work was previously isolated from a mutant screening by Orea et al. [32]. The L. japonicus LjNSE1 mutant seeds were identified by Credali et al. [15] using a TILLING platform (Targeted Induced Local Lesions IN Genomes) developed at the John Innes Centre (Norwich, UK). No endangered plant species were used in this work.

Consent for publication

Not applicable.

\section{Competing interests}

The authors declare that they have no competing interests. 


\section{Publisher's Note}

Springer Nature remains neutral with regard to jurisdictional claims in published maps and institutional affiliations.

Received: 22 November 2016 Accepted: 8 October 2017

Published online: 12 October 2017

\section{References}

1. do Amarante L, Lima JD, Sodek L. Growth and stress conditions cause similar changes in xylem amino acids for different legume species. Environ Exp Bot. 2006;58(1-3):123-9.

2. Waterhouse RN, Smyth AJ, Massonneau A, Prosser IM, Clarkson DT. Molecular cloning and characterisation of asparagine synthetase from Lotus japonicus: dynamics of asparagine synthesis in N-sufficient conditions. Plant Mol Biol. 1996:30(5):883-97.

3. Gaufichon L, Reisdorf-Cren M, Rothstein SJ, Chardon F, Suzuki A. Biological functions of asparagine synthetase in plants. Plant Sci. 2010;179(3):141-53.

4. Duff SMG. Asparagine synthetase. In: Mello JPF, editor. Amino acids in higher plants. CAB international; 2015. p. 100-28.

5. Lam HM, Hsieh MH, Coruzzi G. Reciprocal regulation of distinct asparagine synthetase genes by light and metabolites in Arabidopsis thaliana. Plant J. 1998;16(3):345-53.

6. Herrera-Rodríguez MB, Maldonado JM, Pérez-Vicente R. Light and metabolic regulation of HAS1, HAS1.1 and HAS2, three asparagine synthetase genes in Helianthus annuus. Plant Physiol Biochem. 2004:42(6):511-8.

7. Wong HK, Chan HK, Coruzzi GM, Lam HM. Correlation of ASN2 gene expression with ammonium metabolism in Arabidopsis. Plant Physiol. 2004; 134(1):332-8.

8. Antunes F, Aguilar M, Pineda M, Sodek L. Nitrogen stress and the expression of asparagine synthetase in roots and nodules of soybean (Glycine max). Physiol Plant. 2008;133(4):736-43.

9. Gaufichon L, Masclaux-Daubresse C, Tcherkez G, Reisdorf-Cren M, Sakakibara Y, Hase T, Clément G, Avice JC, Grandjean O, Marmagne A, et al. Arabidopsis thaliana ASN2 encoding asparagine synthetase is involved in the control of nitrogen assimilation and export during vegetative growth. Plant Cell Environ. 2013;36(2):328-42.

10. Gaufichon L, Rothstein SJ, Suzuki A. Asparagine metabolic pathways in Arabidopsis. Plant Cell Physiol. 2016;57(4):675-89.

11. Ohashi M, Ishiyama K, Kojima S, Konishi N, Nakano K, Kanno K, Hayakawa T, Yamaya T. Asparagine synthetase1, but not asparagine synthetase2, is responsible for the biosynthesis of asparagine following the supply of ammonium to rice roots. Plant Cell Physiol. 2015;56(4):769-78.

12. Cánovas FM, Ávila C, Cantón FR, Cañas RA, De la Torre F. Ammonium assimilation and amino acid metabolism in conifers. J Exp Bot. 2007;58(9): 2307-18.

13. Cañas RA, de la Torre F, Cánovas FM, Cantón FR. Coordination of PSAS1 and PSASPG expression controls timing of re-allocated $\mathrm{N}$ utilization in hypocotyls of pine seedlings. Planta. 2007:225(5):1205-19.

14. Credali A, Díaz-Quintana A, García-Calderón M, De la Rosa MA, Márquez AJ, Vega JM. Structural analysis of $\mathrm{K}^{+}$-dependence in L-asparaginases from Lotus japonicus. Planta. 2011;234(1):109-22.

15. Credali A, García-Calderón M, Dam S, Perry J, Díaz-Quintana A, Parniske M, Wang TL, Stougaard J, Vega JM, Márquez AJ. The $\mathrm{K}^{+}$-dependent asparaginase, NSE1, is crucial for plant growth and seed production in Lotus japonicus. Plant Cell Physiol. 2013:54(1):107-18.

16. Zhang Q, Lee J, Pandurangan S, Clarke M, Pajak A, Marsolais F. Characterization of Arabidopsis serine:glyoxylate aminotransferase, AGT1, as an asparagine aminotransferase. Phytochemistry. 2013:85:30-5.

17. Sodek L, Lea PJ, Miflin BJ. Distribution and properties of a potassiumdependent asparaginase isolated from developing seeds of Pisum sativum and other plants. Plant Physiol. 1980;65(1):22-6.

18. Bruneau L, Chapman R, Marsolais F. Co-occurrence of both L-asparaginase subtypes in Arabidopsis: At3g16150 encodes a $\mathrm{K}^{+}$-dependent L-asparaginase. Planta. 2006;224(3):668-79.

19. Bejger M, Imiolczyk B, Clavel D, Gilski M, Pajak A, Marsolais F, Jakolski M. Na ${ }^{+} / \mathrm{K}^{+}$exchange switches the catalytic apparatus of potassium-dependent Lasparaginase. Acta Crystallogr D Biol Cristallogr. 2014;D70:1854-72.

20. Ireland RJ, Joy KW. Subcellular localization of asparaginase and asparagine aminotransferase in Pisum sativum leaves. Plant Physiol. 1983a;72(4):1127-9.

21. Ireland RJ, Joy KW. Purification and properties of an asparagine aminotransferase from Pisum sativum leaves. Arch Biochem Biophys. 1983b;223(1):291-6.
22. Murray AJS, Blackwell RD, Joy KW, Lea PJ, Photorespiratory N. Donors, aminotransferase specificity and photosynthesis in a mutant of barley deficient in serine:glyoxylate aminotransferase activity. Planta. 1987;172(1): 106-13.

23. Havir EA, McHale NAA. Mutant of Nicotiana sylvestris lacking serine: glyoxylate aminotransferase: substrate specificity of the enzyme and fate of $\left[2-{ }^{14} \mathrm{C}\right]$ glycolate in plants with genetically altered enzyme levels. Plant Physiol. 1988;87(4):806-8.

24. Bauwe H, Hagemann M, Fernie AR. Photorespiration: players, partners and origin. Trends Plant Sci. 2010;15(6):330-6.

25. Zhang Q, Marsolais F. Identification and characterization of omega-amidase as an enzyme metabolically linked to asparagine transamination in Arabidopsis. Phytochemistry. 2014:99:36-43.

26. Ta TC, Joy KW, Ireland RJ. Role of asparagine in the photorespiratory nitrogen metabolism of pea leaves. Plant Physiol. 1985;78(2):334-7.

27. O'Leary B, Preston GM, Sweetlove L. Increased $\beta$-cyanoalanine nitrilase activity improves cyanide tolerance and assimilation in Arabidopsis. Mol Plant. 2014;7(1):231-43.

28. Betti M, Arcondéguy T, Márquez AJ. Molecular analysis of two mutants from Lotus japonicus deficient in plastidic glutamine synthetase: functional properties of purified GLN2 enzymes. Planta. 2006;224(5):1068-79.

29. Betti M, García-Calderón M, Pérez-Delgado CM, Credali A. Pal'ove-Balang P, Estivill G, Repcak M, Vega JM, Galván F, Márquez AJ. Reassimilation of ammonium in Lotus japonicus. J Exp Bot. 2014;65(19):5557-66.

30. Handberg K, Stougaard J. Lotus japonicus, an autogamous, diploid legume species for classical and molecular genetics. Plant J. 1992;2(4):487-96.

31. García-Calderón M, Chiurazzi M, Espuny MR, Márquez AJ. Photorespiratory metabolism and nodule function: behavior of Lotus japonicus mutants deficient in plastid glutamine synthetase. Mol Plant-Microbe Interact. 2012;25(2):211-9.

32. Orea A, Pajuelo P, Pajuelo E, Quidiello C, Romero JM, Márquez AJ. Isolation of photorespiratory mutants from Lotus japonicus deficient in glutamine synthetase. Physiol Plant. 2002;115(3):352-61.

33. Márquez AJ, Betti M, García-Calderón M, Pal'ove-Balang P, Díaz P, Monza J. Nitrate assimilation in Lotus japonicus. J Exp Bot. 2005:56(417):1741-9.

34. Sánchez DH, Lippold F, Redestig H, Hannah MA, Erban A, Krämer U, Kopka J Udvardi MK. Integrative functional genomics of salt acclimatization in the model legume Lotus japonicus. Plant J. 2008;53(6):973-87.

35. Pérez-Delgado CM, Moyano TC, García-Calderón M, Canales J, Gutiérrez RA, Márquez AJ, Betti M. Use of transcriptomics and co-expression networks to analyze the interconnections between nitrogen assimilation and photorespiratory metabolism. J Exp Bot. 2016;67(10):3095-108.

36. Czechowski T, Stitt M, Altmann T, Udvardi MK, Scheible WR. Genome-wide identification and testing of superior reference genes for transcript normalization in Arabidopsis. Plant Physiol. 2005;139(1):5-17.

37. Reid DE, Heckmann AB, Novák O, Kelly S, Stougaard JCYTOKININOXIDASE. DEHYDROGENASE3 maintains cytokinin homeostasis during root and nodule development in Lotus japonicus. Plant Physiol. 2016;170(2):1060-74.

38. Saeed Al, Bhagabati NK, Braisted JC, Liang W, Sharov V, Howe EA, Li J, Thiagarajan M, White JA, Quackenbush J. TM4 microarray software suite. Methods Enzymol. 2006;411:134-93

39. Pérez-Delgado CM, García-Calderón M, Sánchez DH, Udvardi MK, Kopka J, Márquez AJ, Betti M. Transcriptomic and metabolic changes associated with photorespiratory ammonium accumulation in the model legume Lotus japonicus. Plant Physiol. 2013;162(4):1834-48.

40. Høgslund N, Radutoiu S, Krussell L, Voroshilova V, Hannah MA, Goffard N, Sanchez DH, Lippold F, Ott T, Sato S, et al. Dissection of symbiosis and organ development by integrated transcriptome analysis of Lotus japonicus mutant and wild-type plants. PLoS One. 2009;4(8):e6556.

41. Díaz P, Betti M, Sánchez DH, Udvardi MK, Monza J, Márquez AJ. Deficiency in plastidic glutamine synthetase alters proline metabolism and transcriptomic response in Lotus japonicus under drought stress. New Phytol. 2010;188(4):1001-13.

42. Sánchez DH, Pieckenstain FL, Szymanski J, Erban A, Bromke M, Hannah MA, Kraemer U, Kopka J, Udvardi MK. Comparative functional genomics of salt stress in related model and cultivated plants identifies and overcomes limitations to translational genomics. PLoS One. 2011;6(2):e17094.

43. Betti M, Pérez-Delgado C, García-Calderón M, Díaz P, Monza J, Márquez AJ. Cellular stress following water deprivation in the model legume Lotus japonicus. Cell. 2012:1(4):1089-06.

44. ArrayExpress database. https://www.ebi.ac.uk/arrayexpress/ Accessed.

45. Gautier L, Cope L, Bolstad BM, Irizarry RA. Affy-analysis of Affymetrix GeneChip data at the probe level. Bioinformatics. 2004;20(3):307-15. 
46. Shamir R, Maron-Katz A, Tanay A, Linhart C, Steinfeld I, Sharan R, Shiloh Y, Elkon R. EXPANDER-an integrative program suite for microarray data analysis. BMC Bioinformatics. 2005;6:232.

47. Ulitsky I, Maron-Katz A, Shavit S, Sagir D, Linhart C, Elkon R, Tanay A, Sharan R, Shiloh Y, Shamir R. Expander: from expression microarrays to networks and functions. Nat Protoc. 2010;5(2):303-22.

48. Kazusa. www.kazusa.or.jp/lotus/ Accessed.

49. Prell J, Poole P. Metabolic changes of rhizobia in legume nodules. Trends Microbiol. 2006;14(4):161-8

50. Lam HM, Coschigani KT, Oliveira IC, Melo-Oliveira R, Coruzzi GM. The molecular-genetics of nitrogen assimilation into amino acids in higher plants. Annu Rev Plant Physiol Plant Mol Biol. 1996;47:569-93.

51. Modde K, Timm S, Florian A, Michl K, Fernie AR, Bauwe H. High serine: glyoxylate aminotransferase activity lowers leaf daytime serine levels, inducing the phosphoserine pathway in Arabidopsis. J Exp Bot. 2017;68(3): 643-56.

52. Pérez-Delgado CM, García-Calderón M, Márquez AJ, Betti M. Reassimilation of photorespiratory ammonium in Lotus japonicus plants deficient in plastidic glutamine synthetase. PLoS One. 2015;10(6):e0130438.

53. Igarashi $\mathrm{D}$, Ishizaki T, Totsuka K, Ohsumi C. ASN2 is a key enzyme in asparagine biosynthesis under ammonium sufficient conditions. Plant Biotechnol. 2009:26(1):153-9.

Submit your next manuscript to BioMed Central and we will help you at every step:

- We accept pre-submission inquiries

- Our selector tool helps you to find the most relevant journal

- We provide round the clock customer support

- Convenient online submission

- Thorough peer review

- Inclusion in PubMed and all major indexing services

- Maximum visibility for your research

Submit your manuscript at www.biomedcentral.com/submit
Biomed Central 